\title{
Pediatric asthma comprises different phenotypic clusters with unique nasal microbiotas
}

\author{
Marcos Pérez-Losada ${ }^{1,2,3^{*}}$ (D), Kayla J Authelet ${ }^{4}$, Claire E Hoptay ${ }^{4}$, Christine Kwak ${ }^{4}$, Keith A Crandall ${ }^{1,2}$ \\ and Robert J Freishtat ${ }^{4}$
}

\begin{abstract}
Background: Pediatric asthma is the most common chronic childhood disease in the USA, currently affecting $\sim 7$ million children. This heterogeneous syndrome is thought to encompass various disease phenotypes of clinically observable characteristics, which can be statistically identified by applying clustering approaches to patient clinical information. Extensive evidence has shown that the airway microbiome impacts both clinical heterogeneity and pathogenesis in pediatric asthma. Yet, so far, airway microbiotas have been consistently neglected in the study of asthma phenotypes. Here, we couple extensive clinical information with 165 rRNA high-throughput sequencing to characterize the microbiota of the nasal cavity in 163 children and adolescents clustered into different asthma phenotypes.
\end{abstract}

Results: Our clustering analyses identified three statistically distinct phenotypes of pediatric asthma. Four core OTUs of the pathogenic genera Moraxella, Staphylococcus, Streptococcus, and Haemophilus were present in at least $95 \%$ of the studied nasal microbiotas. Phyla (Proteobacteria, Actinobacteria, and Bacteroidetes) and genera (Moraxella, Corynebacterium, Dolosigranulum, and Prevotella) abundances, community composition, and structure varied significantly $(0.05<P \leq 0.0001)$ across asthma phenotypes and one of the clinical variables (preterm birth). Similarly, microbial networks of co-occurrence of bacterial genera revealed different bacterial associations across asthma phenotypes.

Conclusions: This study shows that children and adolescents with different clinical characteristics of asthma also show different nasal bacterial profiles, which is indicative of different phenotypes of the disease. Our work also shows how clinical and microbial information could be integrated to validate and refine asthma classification systems and develop biomarkers of disease.

Keywords: $16 \mathrm{~S}$ rRNA, Asthma, Microbiome, Nose, Phenotype

\section{Background}

Pediatric asthma is the most common chronic childhood disease and a major public health problem in the USA, currently affecting $9.3 \%$ of the children $(\sim 7.0$ million), and prevalence continues to rise [1-3]. Prevalence is particularly high (13.9\%) in Washington, DC, mainly

\footnotetext{
* Correspondence: mlosada323@gmail.com

'Computational Biology Institute, Milken Institute School of Public Health," George Washington University, Innovation Hall, Suite 305, 45085 University Drive, Ashburn, VA 20147, USA

${ }^{2}$ Department of Epidemiology and Biostatistics, Milken Institute School of Public Health, George Washington University, Washington, DC 20052, USA Full list of author information is available at the end of the article
}

among African Americans [2], who nonetheless still represent one of the least studied ethnicities with regards to asthma [4]. Childhood asthma is the third leading cause of hospitalization (137,000 cases) among US children, accounting annually for 640,000 emergency department visits $[5,6]$. It is also a major cause of school absenteeism ( 14.4 million lost school days/year) [7], and treatment cost is estimated at $\$ 3.2$ billion/year $[8,9]$.

Pediatric asthma is recognized as a complex condition with differences in severity, natural history, comorbidities, and treatment response [10-13]. A longstanding debate is whether pediatric asthma (and asthma in general)

(c) The Author(s). 2018 Open Access This article is distributed under the terms of the Creative Commons Attribution 4.0 International License (http://creativecommons.org/licenses/by/4.0/), which permits unrestricted use, distribution, and 
is a single disease with a variable presentation or several diseases that have variable airflow obstruction as a common feature. Wenzel [14] proposed that the different phenotypes expressed by patients with asthma are partly dependent on different disease processes in each individual. Thus, the diagnostic label "asthma" likely encompasses many different disease variants with different etiologies and pathophysiologies [10, 11, 15]. Typically, a child's asthma is described in terms of disease phenotypes, which summarizes observable characteristics (clinical, physiological, morphologic, and biochemical), as well as the response to different treatments; thus, they are clinically relevant in terms of presentation, triggers, and treatment response. Asthma phenotypes have long been described by clinicians from their own practice experience, but now are better characterized by applying clustering approaches to patient clinical information collected from cohorts of asthmatic patients (i.e., asthma phenotypic clusters) [16-19]. Using hierarchical clustering, for example, our group has distinguished in a previous study three phenotypic clusters of pediatric asthma in a cohort of children and adolescents from the Washington, DC area (The AsthMaP Project) [18].

The application of novel culture-independent techniques of Next-Generation Sequencing (NGS) has already demonstrated that bacterial communities living in the respiratory airways play a significant role in the onset, development, and severity of asthma [20-29]. Moreover, microbiome research has also shown that the nose is a major reservoir for opportunistic pathogens [30, 31], which can from there spread to other sections of the respiratory tract and potentially cause asthma, but also otitis media or pneumonia, or invade the bloodstream to cause sepsis and meningitis [27, 28, 32-36].

The relationship between airway microbiota and asthma phenotypes is still poorly understood [37-39]. A few studies have explored the interaction in adults $[37,38]$ and have shown significant variation in microbial diversity and the abundance of pathogenic taxa across asthma phenotypes. Whether differences in the composition of microbial populations (pathogenic and commensal) could contribute to asthma in children and adolescents remains to be determined. Defining the relationships between pediatric asthma phenotypes and nasal airway microbiota could ultimately inform our understanding of asthma pathophysiology and could help identify prognostic markers $[18,37]$.

Here, we first applied cluster analysis to clinical, physiological, and biochemical information collected from 163 children and adolescents from Washington, DC belonging to a new cohort (AsthMaP-2) to define phenotypes of pediatric asthma. Then we generated $16 \mathrm{~S} \mathrm{rRNA}$ microbial profiles for those same patients (i) to assess if phenotypic clusters of asthma were associated with nasal bacterial diversity, (ii) to identify bacterial taxa that discriminate among asthma phenotypes, and (iii) to determine the contribution of clinical characteristics to variation in the composition and structure of the nasal microbiome.

\section{Methods \\ Cohort}

AsthMaP-2 is an ongoing study of urban children and adolescents, mainly African Americans (81\% of the subjects in this study with Whites and undetermined accounted for $2 \%$ and $17 \%$, respectively), designed to find associations among airway microbes, environmental exposures, allergic sensitivities, genetics, and asthma. AsthMaP-2 represents a unique sample of otherwise healthy children recruited from the metropolitan Washington, DC area with physician-diagnosed asthma present for at least 1 year. Subjects were enrolled from all the sites in our citywide pediatric and adolescent health system. They were evaluated in our Clinical Research Center via parental interviews, aeroallergen skin testing, nasal sampling, and blood collection. Participants were enrolled between 2013 and 2015 at one study visit at least 4 weeks after completion of their most recent oral steroid dose (baseline) and then followed for 1 year. Individuals who reported a medical history of chronic or complex cardiorespiratory disease were ineligible.

\section{Sample collection}

A total of 205 nasal washes were collected from 163 children and adolescents (ages 6 to 18 years) enrolled in the AsthMaP-2 study at Children's National Medical Center (Washington, DC). Forty-two of those patients came back for an additional visit (5.5 to 6.5 months apart) and one additional sample (E3) was taken (Additional file 1: Table S1). Washes were procured by instilling $5 \mathrm{ml}$ of isotonic sterile saline buffer into each nare, holding it for $10 \mathrm{~s}$ and then blowing into a specimen collection container. Washes were kept in ice while being collected and then stored at $-80^{\circ} \mathrm{C}$ until needed.

\section{High-throughput sequencing}

Total DNA was extracted using the QIAGEN QIAamp DNA Kit (Catalog \# 51304). Before adding the ATL buffer, samples were pre-incubated in $100 \mathrm{uL}$ of lysozyme-TE buffer $\mathrm{pH}=8.0$ for $30 \mathrm{~min}$ at $37^{\circ} \mathrm{C}$. All extractions yielding $>2 \mathrm{ng} / \mu$ of total DNA, as indicated by NanoDrop 2000 UV-Vis Spectrophotometer measuring. DNA extractions were prepared for sequencing using the Schloss' MiSeq_WetLab_SOP protocol (09.2015) in Kozich et al. [40]. Each DNA sample was amplified for the V4 region ( $250 \mathrm{bp})$ of the 16S rRNA gene and libraries were sequenced in a single run of the Illumina MiSeq sequencing platform at the University of Michigan Medical School. Negative controls processed as above showed no PCR band on an agarose gel. 


\section{Phenotype cluster analysis}

A total of 163 children and adolescents from the AsthMaP-2 cohort were included in the cluster analysis. We collected information for 29 clinical variables (Additional file 1: Table S1) during their first visit-no new clinical data were collected during the second visit. Those 29 variables were (in alphabetical order) ACT (Asthma Control Test) score, age (years), age of onset of asthma symptoms (years), allergic rhinitis, antihistamine use in the last 2 weeks, beta agonist use in the last 2 weeks, blood eosinophils (\%), BMI (body mass index) percentile, cold in the last 4 weeks, eczema, FEF (forced expiratory flow), $\mathrm{FEV}_{1}$ (forced expiratory volume in one second) change with bronchodilator, $\mathrm{FEV}_{1} / \mathrm{FVC}$ (functional vital capacity), hospital visit for sample collection, inhaled steroid use in the last 2 weeks, ITG (Integrated Therapeutics Group's Child Asthma Short Form) composite score (see Table 1), leukotriene modifier use in the last 2 weeks, meteorological season during sample collection, NAEPP (National Asthma Education and Prevention Program) severity classification, pets, positive on skin allergen test, postFEV1, preFEV1, preterm birth ( $<35$ weeks of gestation), race, respiratory infection in the last 4 weeks, sex, total serum $\mathrm{IgE}(\mathrm{IU} / \mathrm{ml})$, and vitamin D use in the last 2 weeks. The ACT is a patient-driven test developed to identify those with poorly controlled or refractory asthma. Poor ACT scores indicate the patient's symptoms persist despite the use of conventional treatments. The ITG indicates the asthma-related quality of life and is a self-reported functional health questionnaire. It is scaled between 0 and 100. All collected variables were standardized in

Table 1 Varimax rotation of 11 asthma-relevant variables

\begin{tabular}{|c|c|c|c|c|}
\hline \multirow[t]{2}{*}{ Variable } & \multicolumn{4}{|c|}{ Component } \\
\hline & 1 & 2 & 3 & 4 \\
\hline$\overline{A C T}$ score $^{a}$ & 0.798 & & & \\
\hline ITGc_composite score ${ }^{a}$ & 0.984 & & & \\
\hline ITGf_functional limitations score & 0.864 & & & \\
\hline ITGd_daytime symptoms score & 0.848 & & & \\
\hline ITGn—nighttime symptoms score & 0.842 & & & \\
\hline Post-bronchodilator $\mathrm{FEV}_{1}$ (\% predicted) & & & & 0.967 \\
\hline $\begin{array}{l}\text { Post-bronchodilator } \mathrm{FEV}_{1} / \mathrm{FVC} \\
\text { (\% predicted) }\end{array}$ & & & 0.765 & \\
\hline $\mathrm{FEV}_{1}$ change with bronchodilator & & & 0.784 & \\
\hline Age, years ${ }^{a}$ & & -0.551 & & \\
\hline Blood eosinophil, \% ${ }^{\mathrm{a}}$ & & 0.761 & & \\
\hline Total serum lgE, IU/mL & & 0.741 & & \\
\hline
\end{tabular}

Extraction method: principal component analysis. Rotation method: varimax with Kaiser normalization. Bartlett's test of sphericity $<0.001$. KMO measure of sampling adequacy: 0.471 . ACT asthma control test, ITG Integrated

Therapeutics Group's Child Asthma Short Form, FEV forced expiratory volume, FEF forced expiratory flow, lgE immunoglobulin E. a Variable used in cluster analysis. binary fashion for categorical variables or using a $z$ score for continuous variables. As in previous asthma studies $[16,18]$, relevant variables were chosen if they are measured in the clinical evaluation of asthma and describe asthma phenotypes. Additionally, selection of multiple variables representing the same aspect of asthma was avoided. Principal components analysis (PCA) based on Euclidean distances was then carried out on the eleven selected variables in Table 1 to identify key clinical components relevant to asthma diagnosis and assessment. Nine of these 11 asthma-relevant clinical variables were also used in our previous phenotype cluster analysis [18] of the AsthMaP cohort-a different cohort from that studied here.

Principal component factors were identified using a varimax rotation of the eleven variables. As in Benton et al. [18], cluster analysis was performed in two stages using variables representative of the principal components. In the first stage, hierarchical clustering of the variables using between-groups linkage yielded the probable number of clusters present in AsthMaP-2. A k-means cluster analysis was then performed using this estimated number of clusters. This stage was repeated while specifying one more or one less cluster than the estimate to ensure that the most representative model was obtained. Additionally, the k-means cluster analysis was repeated several times within random AsthMaP-2 subpopulations to ensure reproducibility. Differences between clusters were derived using one-way analysis of variance for normally distributed continuous variables, Kruskal-Wallis for nonparametric continuous variables, and chi-square tests for categorical variables. All statistical tests were performed with SPSS Statistics 17.0 (SPSS, Chicago, IL).

\section{Microbiome analyses}

Raw FASTQ files were processed in mothur v1.35.1 [41] as indicated in the MiSeq SOP (www.mothur.org/wiki/ MiSeq_SOP). Default settings were used to minimize sequencing errors [42]. We removed any sequences with ambiguous bases (maxambig $=0$ ). We sequenced both negative controls and mock communities (reference samples with a known composition) to detect contaminating microbial DNA within reagents and measure sequencing error rate. We did not find evidence of contamination and our sequencing error rate was as low as $0.0071 \%$. Clean paired-end sequences were joined into contigs of $\sim 250 \mathrm{bp}$ and then aligned to the SILVA128-based bacterial reference alignment at www.mothur.org. Contigs $>275$ bp were removed. Chimeras were also removed using uchime [43], and non-chimeric sequences were classified using a naïve Bayesian classifier [44]. Sequences were clustered into Operational Taxonomic Units (OTUs) at the 0.03 similarity threshold (species level). A consensus taxonomy was generated based on the classification of sequences clustered 
within an OTU. OTU sequence representatives and taxonomy were then converted to a BIOM file for subsequent analyses and all OTU singletons $(n=1)$ were eliminated. We normalized our samples using the negative binomial distribution as recommended by McMurdie and Holmes [45] and implemented in the Bioconductor package DESeq2 [46]. This approach simultaneously accounts for library size differences and biological variability. Microbial normalized counts generated this way are referred to as taxon abundances throughout the text. Trees for phylogenetic diversity calculations were constructed using FastTree and midpoint rooting [47]. Taxonomic alpha-diversity was estimated using Shannon and ACE indices, while phylogenetic alpha-diversity was calculated by the Faith's phylogenetic diversity index [48]. Beta-diversity was estimated using phylogenetic UniFrac (unweighted and weighted), Bray-Curtis, and Jaccard distances. The dissimilarity between samples was explored using principal coordinates analysis (PCoA). We also carried out a Mantel correlation test and a Procrustes [49] analysis comparing clinical diversity (29 variables) across 163 patients with their microbiome $16 \mathrm{~S}$ rRNA profiles on the basis of Euclidean similarity metrics and using 10,000 permutations for each test.

We used linear mixed-effects (LME) models analysis, as implemented in the lmer4 $R$ package [50], to investigate associations between alpha-diversity indices and taxa (genera and phyla) abundances (response) and asthma phenotypes (predictor), while accounting for non-independence of subjects (random effect). We have included random effects in our LME models to account for the fact of that 42 patients were sampled twice during the study. We also investigated the potential contribution of other clinical characteristics of the AsthMaP-2 cohort to variation in the composition of the microbiome. Hence, 23 out of the 29 variables listed above were included in the initial LME analyses. To avoid redundancy, we did not include the six variables (ACT score, age, BMI percentile, ITG composite score, percentage of blood eosinophils, and sex) used in the phenotype cluster analysis (see the "Results" section below) to identify asthma phenotypes (composite variable). We also tested LME models with random intercepts and random slopes and different orders of factors. Initial LME models including the 23 variables listed above were compared using the function lmerTest, which performs automatic backward elimination of factors. ANOVA type III tests with Satterthwaite approximation for degrees of freedom were also carried out for hypothesis testing. Model assumptions in final LME models were validated using residual versus fit plots and a normal probability plots.

Beta-diversity UniFrac indices were compared using permutational multivariate analysis of variance (adonis) as implemented in the vegan $\mathrm{R}$ package [51]. Adonis models were compared using the Akaike Index Criterion [52]. Significance was determined through 10,000 permutations.

We believe that only statistically relevant factors in the dataset under study should be included in the final LME models to avoid subjectivity of choice and over-parametrization. Our preliminary LME and adonis analyses showed that random slopes did not have a significant impact on any representation of microbial diversity or taxon abundance. Three co-variables (vitamin D use, antihistamine use, and leukotriene modifier use), however, were significantly associated with one microbial diversity index or one taxon abundance in our LME analyses, but none of those tests were significant for the variable of interest (asthma phenotype). Similarly, preterm birth was significantly associated $(P<0.025)$ to several diversity indices and taxon abundances. Hence, our final (most parsimonious) LME and adonis models included one predictor (asthma phenotype) and one co-variable (preterm birth). We found no significant interactions between asthma phenotype and preterm birth.

Differences in microbial abundances for phyla and genera were also estimated using the Wald test with Cook's distance correction for outliers (DESeq2 package) while accounting for preterm birth. We applied the Benjamini-Hochberg method at alpha $=0.05$ to correct for multiple hypotheses testing [53, 54]. All the analyses above were performed in mothur, QIIME [55], R [56], and RStudio [57].

Microbial organisms coexist in complex ecological networks with various symbiotic relationships. Thus, the presence or abundance of certain individual bacteria most likely affects the presence of others because of those ecologic interactions [58]. We, therefore, estimated networks of co-occurrence of bacterial genera to assess similarities and differences among microbial communities in children and adolescents with different asthma phenotypes. Networks were built in MEGAN [59] using the following parameter settings: threshold $=0.01 \%$ (minimum count required for a taxon to be considered present in a sample), minimum prevalence $=10 \%$ and maximum prevalence $=100 \%$ (minimum and maximum percentage of samples in which a taxon can occur, respectively), and probability $=0.9$ and 0.95 (minimum probability that a co-occurrence between two taxa A and $\mathrm{B}$ must attain so as to be represented by an edge in the graph).

\section{Results}

The AsthMaP-2 cohort is comprised of phenotypic clusters Principal component analysis

Eleven key clinical variables relevant to asthma diagnosis and assessment in AsthMaP-2 were selected for PCA (Table 1). Varimax rotation of those variables identified four principal components representing symptoms/impairment, 
airway reactivity, mucosal evidence of allergy, and systemic evidence of allergy (Table 1). The analysis converged in six iterations. About $70 \%$ of the total variance was explained by these four components, while each of them accounted for $28.4 \%, 19.4 \%, 12.4 \%$, and $11.3 \%$ of the variance after rotation sums of squared loadings. Four highly informative variables were selected for cluster analysis: ACT, ITGc, age, and blood eosinophil (* in Table 1). Two additional variables (sex and BMI percentile) known to be important factors in asthma phenotype were also included in the cluster analysis. Additionally, the most informative of the 29 variables representing asthma status and asthma-related quality of life were compared among clusters (Table 2). It should be noted that $P$-values for ACT, ITGc, age, blood eosinophil, sex, and BMI percentile are not true tests of variance since they were used to maximize distances between the clusters.

\section{Cluster analysis}

The cluster of 163 children and adolescents from the AsthMaP-2 cohort using six asthma-relevant clinical variables resulted in a four-cluster best-fit model with distinct asthma phenotypes. One cluster, however, contained only one single individual with mild asthma and was, therefore, eliminated because its microbiome diversity and composition cannot be statistically compared to other larger clusters. The three remaining clusters differed in their clinical characteristics (Additional file 2: Figure S1 and Table 2). Asthma phenotypic cluster 1 (APC1) was predominantly female (69.5\%) with a lower mean ACT score [mean (SE)] [16.2 (0.5)] and ITGc score [mean (IQR)] [51.2 $(41.7,57.3)]$
(Table 2). APC1 also had the latest mean age of onset of asthma of 4.9 (0.5) years and the highest BMI percentile [81.3 (3.0)] but was not statistically different from APC3 and was within the standard error of APC2. One or more positive allergen skin test (\%) was significantly different between the three groups $(P=0.006)$.

Asthma phenotypic cluster 2 (APC2) had the highest positive allergen tests at $46 \%$, with APC1 and APC3 having $35 \%$ and $25 \%$, respectively (Table 2 ). APC2 also had the highest blood eosinophil \% [mean (IQR)] [8.9 (5.3, 11.7)] and highest serum IgE value $[674.9(139,912)]$. Serum IgE was also statistically significantly different between the three phenotypic clusters $(P=0.013)$. APC2 had many of the hallmarks of allergic asthma. APC2 also had the highest proportion of males (65.1\% male), the youngest age of onset of asthma [3.4 (0.3) years], and the highest proportion of subjects currently using inhaled steroids (ICS) (23.9\%).

Asthma phenotypic cluster 3 (APC3) had the lowest mean BMI percentile [53.7 (4.5)] and the best outcomes for post-bronchodilator pulmonary function tests [e.g., post-bronchodilator $\mathrm{FEV}_{1}$, mean (SE): 109.2 (18.6)] (Table 2). This corresponded to the highest mean ACT score [23.1 (0.4)] and highest ITG composite score $[84.2(72.9,95.8)]$. Furthermore, APC3 had the lowest proportion of subjects with positive allergen skin prick tests (25\%). They also had the smallest proportion of patients currently using inhaled corticosteroids.

In our post hoc between-group analyses, APC1 and APC2 were similar to each other in terms of pulmonary function tests, questionnaire results, and other typical

Table 2 Comparison of asthma characteristics in overall cohort and among asthma phenotypic clusters (APC)

\begin{tabular}{|c|c|c|c|c|c|}
\hline Variable & $\begin{array}{l}\text { All } \\
(n=163)\end{array}$ & $\begin{array}{l}\text { APC1 } \\
(n=51)\end{array}$ & $\begin{array}{l}\text { APC2 } \\
(n=63)\end{array}$ & $\begin{array}{l}\text { APC3 } \\
(n=49)\end{array}$ & $P$ value \\
\hline Sex, \% male ${ }^{a}$ & 52.6 & 29.5 & 65.1 & 63.2 & $<0.001$ \\
\hline Age, years $(S E)^{a}$ & $11.0(0.3)$ & $12.7(0.5)$ & $9.1(0.3)$ & $11.6(0.5)$ & $<0.001$ \\
\hline Age of onset of asthma symptoms, years (SE) & $4.1(0.2)$ & $4.9(0.5)$ & $3.4(0.3)$ & $4.3(0.5)$ & 0.0046 \\
\hline BMI percentile (SE) ${ }^{a}$ & $72(2.2)$ & $81.3(3.0)$ & $81.1(2.7)$ & $53.7(4.5)$ & $<0.001$ \\
\hline Pre-bronchodilator $\mathrm{FEV}_{1}, \%$ predicted (SE) & $85.4(1.4)$ & $88(2.0)$ & $84.8(2.6)$ & $83.2(2.6)$ & 0.401 \\
\hline FEV 1 change with bronchodilator (SE) & $5.8(0.4)$ & $10.3(1.6)$ & $8.6(1.6)$ & $14.4(4.4)$ & 0.299 \\
\hline Post-bronchodilator $\mathrm{FEV}_{1}, \%$ predicted (SE) & $99.2(6.0)$ & $96(2.2)$ & $93.6(2.9)$ & $109.2(18.6)$ & 0.518 \\
\hline NAEPP Severity & $3(2,4)$ & $3(2,4)$ & $3(2,4)$ & $3(2,4)$ & 0.3 \\
\hline ACT score ${ }^{a}$ & $20.4(0.3)$ & $16.2(0.5)$ & $21.6(0.3)$ & $23.1(0.4)$ & $<0.001$ \\
\hline ITGc_composite score $(I Q R)^{a}$ & $68.9(54.2,86.5)$ & $51.2(41.7,57.3)$ & $71.3(64.1,79.2)$ & $84.2(72.9,95.8)$ & $<0.001$ \\
\hline Blood eosinophil, \% (IQR) ${ }^{a}$ & $5.8(2.25,5.75)$ & $4.5(1.8,6.3)$ & $8.9(5.3,11.7)$ & $3.2(1.2,4.7)$ & $<0.001$ \\
\hline Total serum lgE (IQR) & $545(99,710)$ & $579(97,751)$ & $675(139,912)$ & $330(75,368)$ & 0.013 \\
\hline Positive skin allergen tests, $\%$ & 73 & 35 & 46 & 25 & 0.006 \\
\hline Beta agonist use, \% & 27 & 11 & 8 & 11 & 0.471 \\
\hline Inhaled steroid use, \% & 54.6 & 17.2 & 23.9 & 13.5 & 0.342 \\
\hline
\end{tabular}

FEV forced expiratory volume, FEF forced expiratory flow, NAEPP National Asthma Education and Prevention Program, ACT asthma control test, ITG Integrated Therapeutics Group's Child Asthma Short Form, IgE immunoglobulin E, SE standard error, IQR interquartile range. 'Variable used in cluster analysis 
indicators of asthma severity than APC3 was to either APC1 or APC2. A Least significant difference (LSD) test showed several of the APC3 variables to be more different from $\mathrm{APC} 1$ and $\mathrm{APC} 2$ than $\mathrm{APC} 1$ and $\mathrm{APC} 2$ were from each other. For example, post-bronchodilator $\mathrm{FEV}_{1} / \mathrm{FVC}$ (\% predicted) and change in $\mathrm{FEV}_{1}$ post-bronchodilator (\% predicted) variables were statistically different between APC3 when compared to APC1 and APC2 $(0.05<P \leq 0.005)$. The LSD test showed that between APC1 and APC2 post-bronchodilator $\mathrm{FEV}_{1} / \mathrm{FVC}$ (\% predicted) and change in $\mathrm{FEV}_{1}$ post-bronchodilator (\% predicted) were not statistically significant $(P>0.2)$.

As in Benton et al. [18], there was no significant correlation between NAEPP (National Asthma Education and Prevention Program) severity score and cluster membership assignment-the NAEPP score classifies asthma severity based on impairment and future risk. The distribution of NAEPP severity scores was heterogeneous within and between the groups.

\section{The taxonomic composition of the nasal microbiome}

We collected 205 nasal washes from all participants during one or two consecutive visits and sequenced the variable region V4 of the $16 \mathrm{~S}$ rRNA using the Illumina MiSeq platform. Longitudinal differences between nasal microbiomes sampled twice in this study have been described in a previous publication by our group [29]. Here, we focus on the comparison of asthma phenotypes. A total of $6,386,235$ sequences ranging from 530 to 160,718 sequences per sample $($ mean $=25,932$; median $=31,152.4$ ) were obtained after quality control analyses. From these data, we identified a total of 8034 OTUs (Additional file 3: Table S2; OTU taxa).

The nasal microbiomes across all 205 samples included sequences that corresponded to five dominant $(>1 \%)$ Phyla: Firmicutes (37.8), Proteobacteria (36.3\%), Actinobacteria (11.1\%), Bacteroidetes (8.2\%), and Fusobacteria (3.5\%) (Fig. 1). Those Phyla comprised 10 dominant (> 1\%) genera: Moraxella (28.3\%), Staphylococcus (17.8\%), Corynebacterium (10.1\%), Dolosigranulum (7.7\%), Prevotella (5.5\%), Streptococcus (5.5\%), Fusobacterium (3.3\%), Haemophilus (3.2\%), Neisseriaceae sp. (1.4\%), and Peptoniphilus (1.2\%) (Fig. 1). All the other detected genera accounted for $<1 \%$ of the total 16S rRNA sequences each. Each of the 205 nasal microbiomes contained 3 to 10 (mean $=7.8$ genera) of the dominant bacterial genera. All these genera are commonly found in the nose of infants and adults with and without asthma, although in different proportions [24-29, 33, 34, 36, 60-67]. Nonetheless, previous studies $[25,68]$ have also revealed that the nose includes microenvironments containing microbiotas with different diversity and structure and that nasal washes may only capture part of that diversity. Hence, the

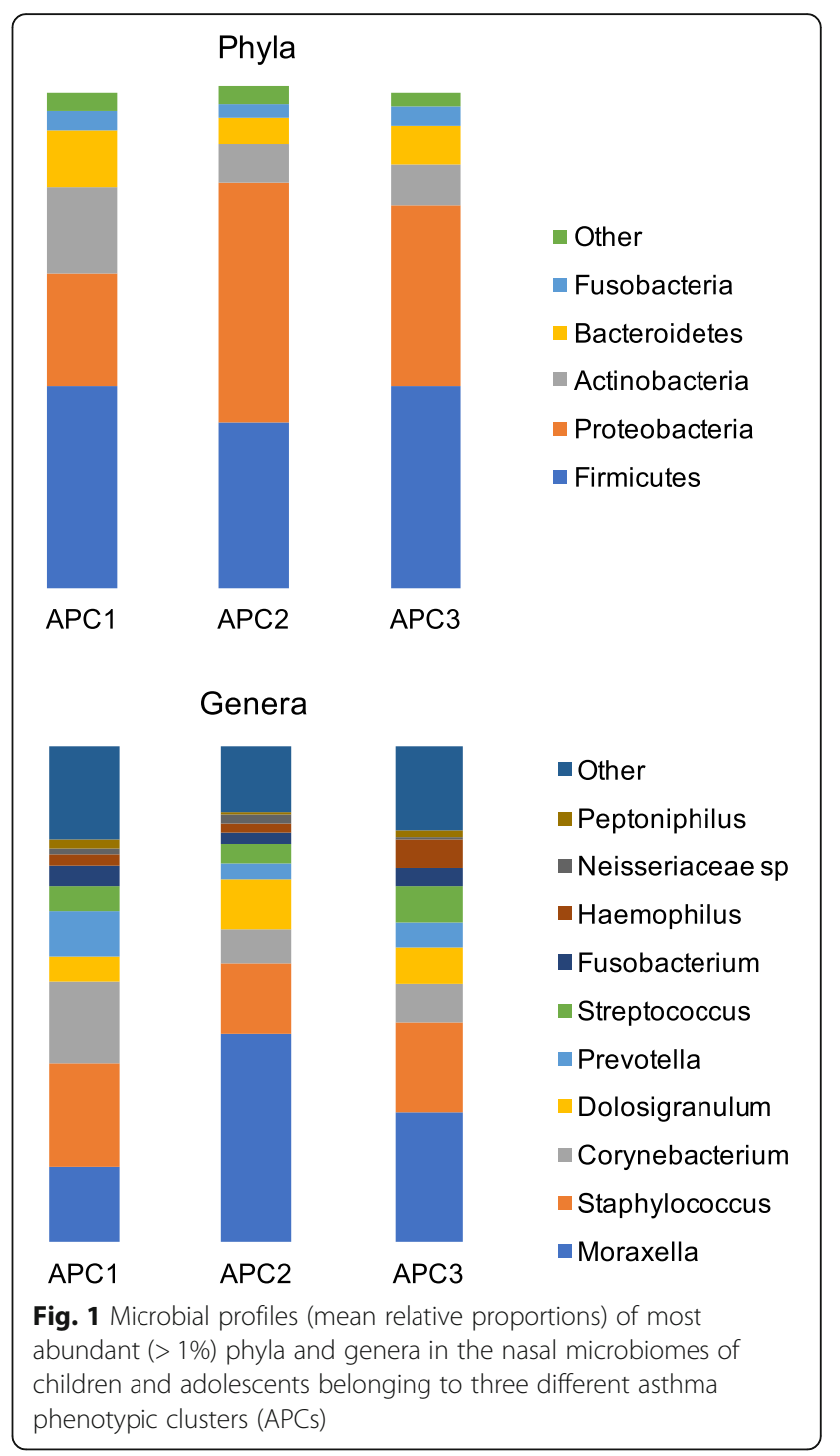

diversity and complexity of the nasal microbiome is probably larger than what we show here.

A variable number of OTUs from these 10 dominant genera were included in the NP core microbiome, which potentially comprises the stable and consistent members and associations from the whole community [69, 70]. The least stringent definition of the core (presence in at least $50 \%$ of the samples) identified 44 OTUs of commensal and pathogenic bacteria; while a more stringent definition (presence in at least 95\% of the samples) included four OTUs of the following genera: Moraxella, Staphylococcus, Streptococcus, and Haemophilus. Pathogenic representatives from these four genera have been consistently associated to asthma $[23,24,27,28,30,31$, $34,36,60]$; hence, these four OTUs may represent fingerprints or biological markers of the NP microbiome in asthmatic children. Future metagenomic studies will need to confirm their consistency across asthmatic cohorts, 
nasal microenvironments $[25,68]$ and specificity (i.e., dominant in asthmatics and absent in healthy controls).

\section{The nasal microbiome of asthmatic children and adolescents varies across phenotypic clusters}

Microbial abundances of all the five dominant $(<1 \%)$ bacterial phyla varied across the three asthma phenotypic clusters (Table 3 and Fig. 1). APC1 showed the highest abundance of Actinobacteria and Bacteroidetes, high abundances of Firmicutes and Fusobacteria, and the lowest abundance of Proteobacteria; APC2 showed the highest abundance of Proteobacteria and the lowest abundance of the other four phyla; and APC3 showed

Table 3 Mean alpha-diversity indices and mean relative proportions of dominant phyla and genera (> 1\%) in decreasing order of abundance for ALL samples and across three asthma phenotypic clusters (APC1, APC2, and APC3) in pediatric asthma

\begin{tabular}{|c|c|c|c|c|c|c|c|}
\hline Taxon & ALL & APC1 & APC2 & APC3 & $F$ & DF & $P(>F)$ \\
\hline \multicolumn{8}{|l|}{ Alpha-diversity } \\
\hline ACE & 225.9 & 227.3 & 217.9 & 234.6 & 0.8 & 151 & 0.4710 \\
\hline PD & 21.5 & 21.5 & 21.5 & 21.6 & 0.02 & 155 & 0.9787 \\
\hline Shannon & 1.82 & 2.02 & 1.59 & 1.88 & 3.1 & 141 & 0.0445 \\
\hline \multicolumn{8}{|l|}{ Beta-diversity } \\
\hline UniFrac-w & - & - & - & - & 5.2 & 2 & 0.0001 \\
\hline UniFrac-unw & - & - & - & - & 1.1 & 2 & 0.1156 \\
\hline Bray-Curtis & - & - & - & - & 3.6 & 2 & 0.0001 \\
\hline Jaccard & - & - & - & - & 2.7 & 2 & 0.0002 \\
\hline \multicolumn{8}{|l|}{ Phyla } \\
\hline Firmicutes & 37.8 & 40.4 & 33.3 & 40.7 & 0.6 & 139 & 0.5548 \\
\hline Proteobacteria & 36.3 & 22.8 & 48.4 & 36.2 & 5.4 & 140 & 0.0056 \\
\hline Actinobacteria & 11.1 & 17.4 & 7.5 & 8.4 & 4.7 & 136 & 0.0105 \\
\hline Bacteroidetes & 8.2 & 11.5 & 5.7 & 7.7 & 3.1 & 153 & 0.0491 \\
\hline Fusobacteria & 3.5 & 4.2 & 2.5 & 4.1 & 1.4 & 126 & 0.2523 \\
\hline \multicolumn{8}{|l|}{ Genus } \\
\hline Moraxella & 28.3 & 15.2 & 41.7 & 25.8 & 6.1 & 141 & 0.0029 \\
\hline Staphylococcus & 17.8 & 20.9 & 14.5 & 18.5 & 0.9 & 142 & 0.4013 \\
\hline Corynebacterium & 10.1 & 16.2 & 6.7 & 7.5 & 4.7 & 136 & 0.0110 \\
\hline Dolosigranulum & 7.7 & 5.3 & 10.0 & 7.4 & 3.0 & 146 & 0.0484 \\
\hline Prevotella & 5.5 & 8.8 & 3.0 & 5.1 & 3.8 & 155 & 0.0242 \\
\hline Streptococcus & 5.5 & 5.2 & 4.3 & 7.4 & 1.1 & 150 & 0.3518 \\
\hline Fusobacterium & 3.3 & 3.9 & 2.4 & 3.7 & 1.1 & 126 & 0.3251 \\
\hline Haemophilus & 3.2 & 2.4 & 1.9 & 5.6 & 1.6 & 113 & 0.2056 \\
\hline Neisseriaceae sp & 1.4 & 1.6 & 1.7 & 0.7 & 1.3 & 156 & 0.2643 \\
\hline Peptoniphilus & 1.2 & 1.8 & 0.6 & 1.2 & 0.9 & 156 & 0.4201 \\
\hline
\end{tabular}

Linear mixed-effects (LME) models results are shown for alpha-diversity indices and taxa abundances, while permutational multivariate analysis of variance (adonis) results are shown for beta-diversity indices. The significance of LME models was estimated using ANOVA of type III with Satterthwaite approximation for degrees of freedom. For each test, we report the relevant $F$ statistic $(F)$, degrees of freedom (DF), and significance $(P(>F))$ intermediate proportions of Proteobacteria, Actinobacteria, and Bacteroidetes and high abundances of Firmicutes and Fusobacteria (as APC1). Our LME analyses showed significant differences in the mean relative proportions of Proteobacteria $(P=0.0005)$, Actinobacteria $(P=0.001)$, and Bacteroidetes $(P=0.0491)$ across the three asthma phenotypic clusters.

Microbial profiles of some of the most abundant bacterial genera (Table 3 and Fig. 1) also varied across asthma phenotypic clusters. APC1 showed the highest abundance of Corynebacterium and Prevotella and the lowest abundance of Moraxella and Dolosigranulum; APC2 showed the highest abundance of Moraxella and the lowest abundance of Corynebacterium, Staphylococcus, and Prevotella; while APC3 showed an intermediate abundance of those five genera. Our LME analyses showed significant associations with asthma phenotypic cluster for Moraxella $(P=0.0003)$, Corynebacterium $(P=0.0014)$, Dolosigranulum $(P=0.0484)$, and Prevotella $(P=0.0256)$. All these significant associations above between phyla and genera and asthma phenotypic clusters were confirmed by the Wald test with Cook' $\mathrm{s}$ distance correction for outliers $(0.05 \leq P \leq 0.0001)$ while accounting for preterm birth.

Alpha-diversity index (Shannon, ACE, and PD) varied across asthma phenotypic clusters (Additional file 4: Figure S2), but only Shannon estimates, which showed less diversity for APC2, were significantly different $(P=0.024)$ in our LME analyses (Table 3).

PCoAs of UniFrac (unweighted and weighted), Bray-Curtis, and Jaccard distances showed partial segregation of the microbiotas from each asthma phenotype (Additional file 5: Figure S3). Our adonis analyses detected significant differences $(P \leq 0.0002)$ in beta-diversity among phenotypes for all of the four distances but UniFrac unweighted $(P=0.1172)$. Mantel $(r=0.54, P=0.038)$ and Procrustes tests showed a significant correlation between asthma phenotypic diversity and nasal microbial diversity (sum of squares $\mathrm{M}^{2}=0.29, P=0.001$ ).

Genus-based microbial interaction networks varied across the three asthma phenotypic clusters (Fig. 2). Only co-occurrence (positive) relationships were detected under our parameter settings. APC1 (red) and APC2 (cyan) involved four genera and five edges, while APC3 (green) involved 6 genera and 9 edges at probability $=0.9$. All phenotype networks involved two genera and one edge at probability $=0.95$. There was partial overlap among all networks, with genera Staphylococcus, Streptococcus, and Corynebacterium being involved in all of them.

Preterm birth was significantly associated to variation in alpha-diversity (Shannon; $P=0.005$ ), all beta-diversity indices $(P \leq 0.0008)$ except UniFrac unweighted distance, and the abundances of four taxa: Proteobacteria 


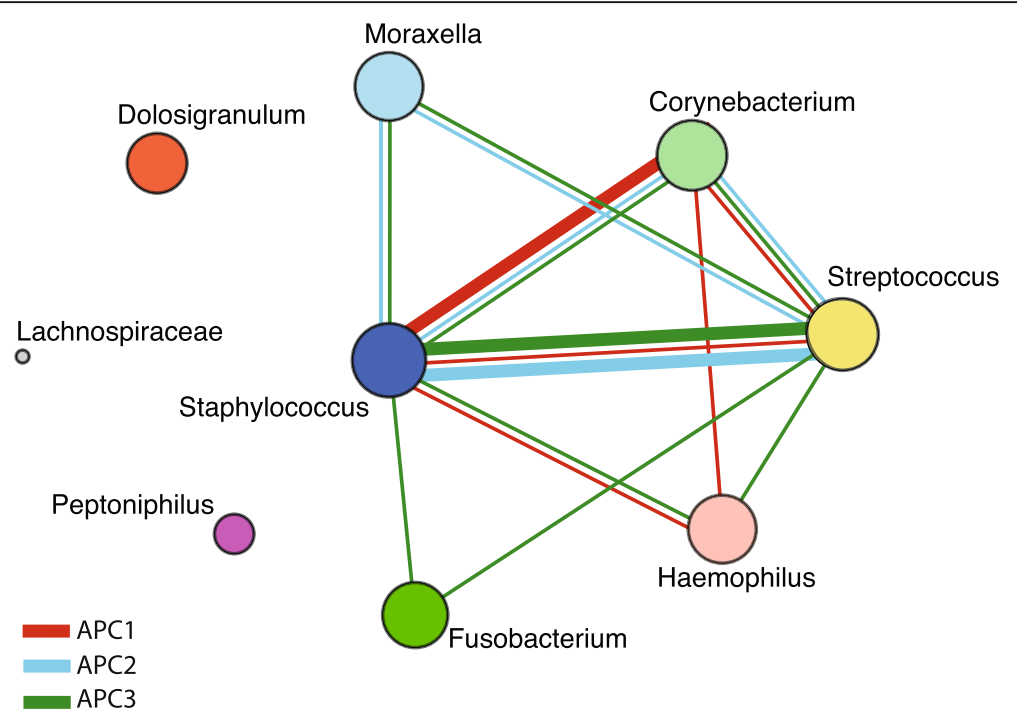

Fig. 2 Network analyses of microbial co-occurrences in the nasal microbiomes of children and adolescents belonging to three different asthma phenotypic clusters (APCs). Different network edge colors were used for each phenotype. Thin edges correspond to probabilities of 0.90, while thick edges correspond to probabilities of 0.95 . Only bacterial genera involved in a network are displayed

$(P=0.0017)$, Firmicutes $(P=0.0233)$, Moraxella $(P=0.0042)$, and Staphylococcus $(P=0.0167)$.

\section{Discussion}

In this study, we investigated the composition and structure of bacterial communities inhabiting the nasal cavity of 163 asthmatic children and adolescents from the Washington, DC area. We first used clinical, physiological, and biochemical information to separate patients into three phenotypic clusters corresponding to different phenotypes of asthma, and then used bacterial $16 \mathrm{~S}$ rRNA sequences to compare their NP microbiotas across those three asthma phenotypic clusters.

\section{Pediatric asthma comprises different phenotypic clusters} Our cluster analysis of the AsthMaP-2 cohort identified three clusters corresponding to three pediatric asthma phenotypes. Clusters of similar proportion and characteristics were identified in a previous study of asthmatic children and adolescents, mainly African Americans, also from the DC area using the AsthMaP cohort [18]. AsthMaP-2 and AsthMaP are two consecutive longitudinal cohorts including different patients. AstMaP-2 is analyzed here for the first time using both clinical and microbiome data. The clinical variables used here to cluster by case overlapped with the 11 variables used to cluster the original AsthMaP cohort, but here, we used only 6 variables. Despite the use of fewer variables to cluster, the k-means cluster analysis still produced three clusters of similar clinical characteristics. Since in both cohorts we found three phenotypic clusters comprised of individuals of similar clinical characteristics, we may conclude that the studied asthma phenotypes are probably stable and reproducible.

Asthma phenotypic cluster 1 (APC1) had lower mean scores in terms of severity measures (ITGc score) and asthma control measures (ACT), as previously mentioned. This predominantly African American female cluster with a mean age of $12.1(0.50)$ years has many of the hallmarks of patients at risk for refractory asthma. Benton et al. [18] previously established a phenotypic cluster of predominantly overweight or obese asthmatics in the AsthMaP cohort. This phenotypic cluster had a mean age of $13.8(0.6)$ years. The potential re-appearance of this phenotypic cluster indicates a possible socio-biological context for this group's disease process. Data from the Childhood Asthma Management Program (CAMP) also suggests that the onset of puberty in both sexes represents a turning point in the disease progression for both females and males. Female symptoms worsen during puberty while male symptoms improve in late puberty [71]. This, in conjunction with our cluster analysis, suggests there may be an association between sex hormones and the pathogenesis of asthma.

APC2 was predominantly male with a preponderance of positive allergic asthma measures (e.g., blood eosinophil \%, serum IgE) and the lowest mean age of $9.1(0.3)$ years. The IgE antibody production in asthma patients mediates hypersensitivity reactions; it binds to receptors in mast cells and basophils and triggers the release of mediators. APC2 high mean IgE values indicate atopy in this phenotypic cluster.

APC3 also responded to bronchodilator use measured by the pulmonary function tests (e.g., post-bronchodilator $\mathrm{FEV}_{1}$ ) and asthma control questionnaires. APC3 was also 
predominantly male and had the lowest mean BMI percentile of 53.7 (0.6). Benton et al. [18] also introduced a similar phenotypic cluster ( $81 \%$ male) with a mean BMI percentile of 51 (3.2) in the AsthMaP cohort. This cluster also had the highest mean ITGc score of the three clusters. Another study of a cohort of African American and Hispanic children in New York City has demonstrated an inverse correlation between asthma risk and extreme BMI percentiles (underweight and overweight, 95th percentile) in young males [72]. This phenotype of low-BMI males with asthma appears in both the AsthMaP and AsthMaP-2 cohorts for the males with the lowest BMI percentiles.

\section{The nose is a reservoir for opportunistic pathogens}

The nose is the major ecological niche for potential pathogens that cause lower respiratory infections such as asthma [23, 24, 27, 28, 30, 31, 34, 36, 60]. All core microbiome genera (Fig. 1) we detected in the nose (Moraxella, Staphylococcus, Streptococcus, and Haemophilus) include opportunistic pathogenic species of the airways [73, 74]. Other population-based microbiome studies of the lower and upper respiratory airways (excluding the nose and nasopharynx) have also shown enrichment of these genera in asthmatic infants, children, or adults [20,34, 75-77]. Thus, our results confirm in children and adolescents the potential role of the nose as a reservoir of pathogens for other sections of the respiratory tract $[23,29,34]$.

\section{The nasal microbiome changes across pediatric asthma phenotypes and preterm birth}

The relationship between airway microbiology and asthma phenotypes is still poorly understood [37-39]. Here, we investigated if unbiased phenotypic clusters of pediatric asthma and clinical, physiological, and biochemical characteristics were associated with nasal bacterial diversity. Microbial profiles of some of the most abundant bacterial phyla and pathogenic genera associated with asthma (e.g., Proteobacteria and Moraxella) varied significantly (LME, $P<0.05$ ) across pediatric asthma phenotypic clusters. Similarly, both alpha-diversity (Shannon index) and beta-diversity (UniFrac weighted, Bray-Curtis, and Jaccard distances) estimates revealed significant differences (Table 3; LME, $P<0.05$ ) in the composition (abundance and evenness) and structure, respectively, of the microbial communities of the three asthma phenotypes. Additionally, microbial co-occurrences (as indicated by our network analysis) among dominant bacterial members also varied across asthma phenotypic clusters (Fig. 2). This likely reflects different symbiotic interactions between pathogenic and commensal bacteria in the nose as seen in other respiratory diseases $[65,78]$. Nonetheless, taxa co-occurrences as described here, do not necessarily reflect functional relationships. Further studies, focusing on the functional capabilities these taxa display within the nose (e.g., via RNASeq analyses, see [24]) are needed to determine if these bacteria truly contribute to symbiosis during asthma.

By combining microbiome and clinical information from the same cohort of individuals, we were able to distinguish different disease variants of pediatric asthma with potentially different etiologies and pathophysiologies. No other study we are aware of has studied the relationships between pediatric asthma phenotypes and airway microbiomes, but a few studies have investigated microbiome-phenotype associations in adult asthma. Taylor et al. [37] found that participants with four different inflammatory asthma phenotypes (as indicated by relative proportions of neutrophils and eosinophils) also showed differences in composition and structure for both pathogens and commensal bacteria. The study also revealed differences in the abundance of opportunistic pathogens, like Moraxella, among inflammatory phenotypes. In our study, Moraxella abundance also varied significantly (LME; $F=5.6 ; \mathrm{DF}=119 ; P=0.0194$ ) in relation to eosinophil proportions across pediatric phenotypes-we did not collect neutrophil information. Future studies will need to assess if microbial profiles seen in adults with different inflammatory asthma phenotypes mimic (or develop from) those seen in asthmatic children and adolescents.

Another study by Zhang et al. [38] also revealed marked differences in the distribution of bacterial phyla (Proteobacteria and Firmicutes) and genera (Streptococcus and Prevotella) between two phenotypes of asthma severity (severe and non-severe) in adults. They did not observe differences in microbial composition (alpha-diversity) between phenotypes, but the opposite was true for microbial community structure (beta-diversity). In our study, asthma severity was classified as mild, moderate, intermediate, and severe, and it was not significantly associated $(P>0.07)$ with microbial diversity or taxa abundances. Several factors may explain the different outcomes between our results and Zhang et al.'s study; firstly, the studied cohorts have different characteristics, including patient age, ethnicity, country of residence, and treatment; secondly, the analyzed sample types were different-we used nasal washes while Zhang et al. used induced sputum; thirdly, the applied asthma severity classification systems are different-we used the National Asthma Education and Prevention Program (NAEPP) [79], while Zhang et al. used the Severe Asthma Protocol [80]. The microbiome similarity seen in our study across asthma severity types is supported by our cluster analysis and agrees with a previous clinical study of 154 children in the AsthMaP project (Washington, DC) [18], which also did not find significant differences in NAEPP severity 
scores and cluster membership assignment. Our study, hence, confirms previous results [18] and suggests that a clustering method like the one used here coupled with microbial profiles could be used as a new strategy to group asthmatic children and adolescents based on their asthma phenotype. Future work is needed to investigate the efficacy of current and new treatment options for these asthma phenotypes.

Preterm birth was significantly associated with variation in microbiome diversity and abundance of several microbial taxa. Individuals born prematurely undergo dramatically different early-life exposures in the neonatal intensive care unit (NICU). Prematurity-related challenges include nosocomial pathogens, supplemental oxygen, mechanical respiratory support, broad-spectrum antimicrobials, and deprivation of the normal intrauterine environment. Previous studies have shown that the airway microbiome may also play a role in post-natal problems faced by premature infants [73, 81]. A recent study has shown that the NP microbiota of premature infants is altered relative to that of infants born at term and that those changes persist during at least early childhood (6 months to 2 years) [82]. Microbial dysbiosis may play an important role in modulating airway inflammatory and immune responses [83]. Indeed, previous studies have established that the early nasal and nasopharyngeal microbial composition correlates with individual frequency and severity of upper and lower respiratory infections as well as subsequent risk of developing asthma [27, 33, 84-86]. Our results suggest that nasal microbiome changes acquired by preterm neonates may persist into adolescence. Future longitudinal studies will be needed to further investigate the interplay between preterm birth, nasal microbiota, and the development of airway immune responses against respiratory pathogens in early life.

\section{Limitations}

Metataxonomic studies like ours suffer from the inherent limitations of collecting sequence data from a single partial gene target (16S rRNA) [87]. First, there is no validation of the composition and structure of the microbiotas using an alternative molecular marker. There are well-known issues with bias in PCR amplification early in the PCR reaction that can impact microbial compositional assessment. The single partial gene approach has also limited resolution at the species and sometimes even genus level for taxonomic assignment. Nevertheless, the composition of the nasal microbiomes in this study is similar to those described in previous microbial studies of the nose and nasopharynx in asthmatics using the same cohort but different individuals $[25,29,60]$ or different cohorts [27, 33, 34]. Second, the relevance of detecting organisms associated with specific phenotypes of clinical variables is unknown-do the taxa that differentiate asthma phenotypic clusters engage in distinct interactions with the host or induce features consistent with the phenotypic features of the cluster in which they are enriched? Previous research by our group [24, 26] has used dual transcriptomics (RNAseq) to investigate host-microbe interactions during asthma. Metatranscriptomic insights coupled with longitudinal sampling (as oppose to the cross-sectional sampling design using here) can help to clarify whether specific microbes are drivers or bystanders in asthmatic patients. Our future microbiome research will address this issue. Lastly, we did not include healthy control samples in our study; hence, we cannot test how bacterial profiles in asthmatics compare to healthy children of similar age. Multiple studies, however (including ours), have already established that the nasal microbiomes of asthmatic and healthy individuals differ across ages in infants, children, and adults $[20,21,29,34,77]$. The focus of our study was different since we aimed to determine if asthmatic children belonging to different asthma phenotypes also have different microbiomes. Our results demonstrate this difference in a rather large cohort; however, including control samples would help to further validate if relative microbial enrichments are exclusive to children with asthma or are also detected in upper airways of healthy children or non-asthmatic individuals with a relevant history of nasal allergies or frequent respiratory infections $[29,77,88]$.

\section{Conclusions}

Microbiome information has been consistently neglected in the study of asthma phenotypes. Our study identifies significant differences in the composition and structure of the nasal microbiotas of children and adolescents across asthma phenotypic clusters. Microbial profiles (e.g., Moraxella and Corynebacterium) coupled with clinical, physiological, and biochemical factors (e.g., ITG and ACT scores) revealed different disease variants of pediatric asthma. This information could ultimately inform our understanding of asthma pathophysiology, validate (and further refine) current asthma classifications, improve current prognostic markers (i.e., asthma biomarkers) of disease $[18,37]$, and custom-fit treatment options for precision medicine. This study represents a step forward towards that ultimate goal.

\section{Additional files}

Additional file 1: Table S1. Clinical and demographic characteristics of the cohort analyzed in this study. (XLSX $70 \mathrm{~kb}$ )

Additional file 2: Figure S1. Heatmap of six clinical variables (ACT score, age, BMI percentile, sex, ITGc and blood eosinophil \%) showing three asthma phenotypic clusters (APCs). (PDF 903 kb) 
Additional file 3: Table S2. Number of clean sequences per OTU and sample and corresponding taxonomic identification up to the genus level. (XLSX $4706 \mathrm{~kb})$

Additional file 4: Figure S2. Box plots of Shannon, ACE and phylogenetic alpha-diversity of microbiotas from children and adolescents belonging to three asthma phenotypic clusters (APCs). (PDF $1885 \mathrm{~kb}$ )

Additional file 5: Figure S3. Principal coordinates analyses of unweighted and weighted UniFrac, Bray-Curtis and Jaccard distances among microbiotas from children and adolescents belonging to three asthma phenotypic clusters (APCs). (PDF $1160 \mathrm{~kb}$ )

\section{Acknowledgements}

We thank Dr. Lynch for her comments on a former version of this manuscript. We also thank the GWU Colonial One High Performance Computing Cluster for computational time.

\section{Funding}

MP-L was funded in part by a K12 Career Development Program K12HL119994 award, Milken Institute School of Public Health Pilot Fund Program, the Margaret Q. Landenberger Research Foundation and the Fundação para a Ciência e a Tegnologia (T495756868-00032862). The AsthMaP-2 project was supported by Award Numbers R01MD007075 to RJF and UL1TR000075 from the NIH National Center for Advancing Translational Sciences (RJF, KAC), in addition to funding to RJF from the Clark Charitable Foundation. Its contents are solely the responsibility of the authors and do not necessarily represent the official views of the National Center for Advancing Translational Sciences or the National Institutes of Health.

\section{Availability of data and materials}

Sequence data have been deposited in GenBank under SRA accession number SRP069020.

\section{Authors' contributions}

$M P-L, K A C$, and RJF conceived and designed the study. MP-L, CEH, and RJF collected and sequenced the samples. KJA, CEH, and RJF analyzed the clinical, physiological, and biochemical data. MP-L and CK carried out the microbiome analyses. MP-L and KJA wrote the manuscript with all authors contributing. All authors read and approved the final manuscript.

\section{Ethics approval and consent to participate}

All participants in this study were part of The AsthMaP-2 Project. AsthMaP-2 and the work presented here was approved by the Children's National Medical Center Institutional Review Board. Written consent was obtained from all independent participants or their legal guardians using the IRB-approved informed consent documents.

\section{Consent for publication}

Not applicable

\section{Competing interests}

The authors declare that they have no competing interests.

\section{Publisher's Note}

Springer Nature remains neutral with regard to jurisdictional claims in published maps and institutional affiliations.

\section{Author details}

'Computational Biology Institute, Milken Institute School of Public Health, George Washington University, Innovation Hall, Suite 305, 45085 University Drive, Ashburn, VA 20147, USA. ${ }^{2}$ Department of Epidemiology and Biostatistics, Milken Institute School of Public Health, George Washington University, Washington, DC 20052, USA. ${ }^{3} \mathrm{CIBIO}-\mathrm{InBIO}$, Centro de Investigação em Biodiversidade e Recursos Genéticos, Universidade do Porto, Campus Agrário de Vairão, 4485-661 Vairão, Portugal. ${ }^{4}$ Division of Emergency Medicine, Children's National Medical Center, Washington, DC 20010, USA.
Received: 5 May 2018 Accepted: 25 September 2018

Published online: 04 October 2018

\section{References}

1. Freishtat RJ, Watson AM, Benton AS, Iqbal SF, Pillai DK, Rose MC, Hoffman EP. Asthmatic airway epithelium is intrinsically inflammatory and mitotically dyssynchronous. Am J Respir Cell Mol Biol. 2011;44(6):863-9.

2. Stemmy EJ, Benton AS, Lerner J, Alcala S, Constant SL, Freishtat RJ. Extracellular cyclophilin levels associate with parameters of asthma in phenotypic clusters. J Asthma. 2011;48(10):986-93.

3. National Institute of Allergy and Infectious Diseases. NIAID Strategic Plan 2013. 2013. Available from: http://www.niaid.nih.gov/about/whoweare/ planningpriorities/Pages/default.aspx. Last accessed: 6 Jan 2015.

4. Barnes KC, Grant AV, Hansel NN, Gao P, Dunston GM. African Americans with asthma: genetic insights. Proc Am Thorac Soc. 2007:4(1):58-68.

5. Ferrante SC, Nadler EP, Pillai DK, Hubal MJ, Wang Z, Wang JM, GordishDressman H, Koeck E, Sevilla S, Wiles AA, et al. Adipocyte-derived exosomal miRNAs: a novel mechanism for obesity-related disease. Pediatr Res. 2015; 77(3):447-54.

6. Koeck ES, lordanskaia T, Sevilla S, Ferrante SC, Hubal MJ, Freishtat RJ, Nadler EP. Adipocyte exosomes induce transforming growth factor beta pathway dysregulation in hepatocytes: a novel paradigm for obesity-related liver disease. J Surg Res. 2014;192(2):268-75.

7. Akinbami LJ, Moorman JE, Liu X. Asthma prevalence, healthcare use, and mortality: United States, 2005-2009. National health statistics report; no 32. Hyattsville: National Center for Health Statistics; 2011.

8. Barnett SB, Nurmagambetov TA. Costs of asthma in the unites states: 20022007. J Allergy Clin Immunol. 2011;127:145-52.

9. von Mutius E, Hartert T. Update in asthma 2012. Am J Respir Crit Care Med. 2013;188(2):150-6.

10. Wenzel SE. Asthma phenotypes: the evolution from clinical to molecular approaches. Nat Med. 2012;18(5):716-25.

11. Borish L, Culp JA. Asthma: a syndrome composed of heterogeneous diseases. Ann Allergy Asthma Immunol. 2008;101(1):1-8.

12. Martinez FD. Definition of pediatric asthma and associated risk factors. Pediatr Pulmonol Suppl. 1997;15:9-12.

13. Cowan K, Guilbert TW. Pediatric asthma phenotypes. Curr Opin Pediatr. 2012;24(3):344-51

14. Wenzel SE. Asthma: defining of the persistent adult phenotypes. Lancet. 2006;368(9537):804-13

15. Aujla SJ, Ross KR, Chmiel JF, Holguin F. Airway molecular phenotypes in pediatric asthma. Curr Opin Allergy Clin Immunol. 2011;11(2):122-6.

16. Haldar P, Pavord ID, Shaw DE, Berry MA, Thomas M, Brightling CE, Wardlaw AJ, Green RH. Cluster analysis and clinical asthma phenotypes. Am J Respir Crit Care Med. 2008;178(3):218-24.

17. Moore WC, Meyers DA, Wenzel SE, Teague WG, Li H, Li X, D'Agostino R Jr, Castro M, Curran-Everett D, Fitzpatrick AM, et al. Identification of asthma phenotypes using cluster analysis in the Severe Asthma Research Program. Am J Respir Crit Care Med. 2010;181(4):315-23.

18. Benton AS, Wang Z, Lerner J, Foerster M, Teach SJ, Freishtat RJ. Overcoming heterogeneity in pediatric asthma: tobacco smoke and asthma characteristics within phenotypic clusters in an African American cohort. J Asthma. 2010;47(7):728-34.

19. Moore WC, Bleecker ER, Curran-Everett D, Erzurum SC, Ameredes BT, Bacharier L, Calhoun WJ, Castro M, Chung KF, Clark MP, et al. Characterization of the severe asthma phenotype by the National Heart, Lung, and Blood Institute's Severe Asthma Research Program. J Allergy Clin Immunol. 2007:119(2):405-13.

20. Huang YJ, Boushey HA. The microbiome and asthma. Ann Am Thorac Soc. 2014;11 Suppl 1:S48-51.

21. Huang YJ, Boushey HA. The microbiome in asthma. J Allergy Clin Immunol. 2015;135(1):25-30

22. Brar T, Nagaraj S, Mohapatra S. Microbes and asthma: the missing cellular and molecular links. Curr Opin Pulm Med. 2012;18(1):14-22.

23. Dickson RP, Huffnagle GB. The lung microbiome: new principles for respiratory bacteriology in health and disease. PLoS Pathog. 2015;1 (7): e1004923.

24. Pérez-Losada M, Castro-Nallar E, Bendall ML, Freishtat RJ, Crandall KA. Dual Transcriptomic profiling of host and microbiota during health and disease in pediatric asthma. PLoS One. 2015;10(6):e0131819. 
25. Pérez-Losada M, Crandall KA, Freishtat RJ. Two sampling methods yield distinct microbial signatures in the nasopharynges of asthmatic children. Microbiome. 2016;4:25.

26. Castro-Nallar E, Shen Y, Freishtat RJ, Pérez-Losada M, Manimaran S, Liu G, Spira A, Johnson WE, Crandall KA. Integrating metagenomics and host gene expression to characterize asthma-associated microbial communities. BMC Med Genet. 2015;8:50.

27. Teo SM, Mok D, Pham K, Kusel M, Serralha M, Troy N, Holt BJ, Hales BJ, Walker ML, Hollams E, et al. The infant nasopharyngeal microbiome impacts severity of lower respiratory infection and risk of asthma development. Cell Host Microbe. 2015;17:704-15.

28. Bogaert D, Keijser B, Huse S, Rossen J, Veenhoven R, van Gils E, Bruin J, Montijn R, Bonten M, Sanders E. Variability and diversity of nasopharyngeal microbiota in children: a metagenomic analysis. PLoS One. 2011;6(2):e17035.

29. Pérez-Losada M, Alamri L, Crandall KA, Freishtat RJ. Nasopharyngeal microbiome diversity changes over time in children with asthma. PLoS One. 2017;12(1):e0170543.

30. Esposito S, Principi N. Impact of nasopharyngeal microbiota on the development of respiratory tract diseases. Eur J Clin Microbiol Infect Dis. 2018;37(1):1-7.

31. Huang YJ. Nasopharyngeal microbiota: gatekeepers or fortune tellers of susceptibility to respiratory tract infections? Am J Respir Crit Care Med. 2017;196(12):1504-5.

32. Garcia-Rodriguez JA, Fresnadillo Martinez MJ. Dynamics of nasopharyngeal colonization by potential respiratory pathogens. J Antimicrob Chemother. 2002;50 Suppl S2:59-73.

33. Biesbroek G, Tsivtsivadze E, Sanders EA, Montijn R, Veenhoven RH, Keijser BJ, Bogaert D. Early respiratory microbiota composition determines bacterial succession patterns and respiratory health in children. Am J Respir Crit Care Med. 2014;190(11):1283-92.

34. Hilty M, Burke C, Pedro H, Cardenas P, Bush A, Bossley C, Davies J, Ervine A, Poulter L, Pachter $L$, et al. Disordered microbial communities in asthmatic airways. PLoS One. 2010;5(1):e8578.

35. Dickson RP, Erb-Downward JR, Huffnagle GB. The role of the bacterial microbiome in lung disease. Expert Rev Respir Med. 2013;7(3):245-57.

36. Prevaes SM, de Winter-de Groot KM, Janssens HM, de Steenhuiisen Piter WA, Tramper-Stranders GA, Wyllie AL, Hasrat R, Tiddens HA, van Westreenen $M$, van der Ent CK, et al. Development of the nasopharyngeal microbiota in infants with cystic fibrosis. Am J Respir Crit Care Med. 2016;193(5):504-15.

37. Taylor SL, Leong LEX, Choo JM, Wesselingh S, Yang IA, Upham JW, Reynolds PN, Hodge S, James AL, Jenkins $C$, et al. Inflammatory phenotypes in patients with severe asthma are associated with distinct airway microbiology. J Allergy Clin Immunol. 2018;141(1):94-103.e15.

38. Zhang Q, Cox M, Liang Z, Brinkmann F, Cardenas PA, Duff R, Bhavsar P, Cookson W, Moffatt M, Chung KF. Airway microbiota in severe asthma and relationship to asthma severity and phenotypes. PLoS One. 2016;11(4):e0152724.

39. Chung KF. Potential Role of the Lung Microbiome in Shaping Asthma Phenotypes. Ann Am Thorac Soc. 2017;14(Supplement_5):S326-31.

40. Kozich JJ, Westcott SL, Baxter NT, Highlander SK, Schloss PD. Development of a dual-index sequencing strategy and curation pipeline for analyzing amplicon sequence data on the MiSeq Illumina sequencing platform. Appl Environ Microbiol. 2013;79(17):5112-20.

41. Schloss PD, Westcott SL, Ryabin T, Hall JR, Hartmann M, Hollister EB, Lesniewski RA, Oakley BB, Parks DH, Robinson CJ, et al. Introducing mothur: open-source, platform-independent, community-supported software for describing and comparing microbial communities. Appl Environ Microbiol. 2009;75(23):7537-41.

42. Schloss PD, Gevers D, Westcott SL. Reducing the effects of PCR amplification and sequencing artifacts on 165 rRNA-based studies. PLoS One. 2011;6(12):e27310

43. Edgar RC, Haas BJ, Clemente JC, Quince C, Knight R. UCHIME improves sensitivity and speed of chimera detection. Bioinformatics. 2011:27(16):2194-200.

44. Wang Q, Garrity GM, Tiedje JM, Cole JR. Naive Bayesian classifier for rapid assignment of rRNA sequences into the new bacterial taxonomy. Appl Environ Microbiol. 2007;73(16):5261-7

45. McMurdie PJ, Holmes S. Waste not, want not: why rarefying microbiome data is inadmissible. PLoS Comput Biol. 2014;10(4):e1003531.

46. Love Ml, Huber W, Anders S. Moderated estimation of fold change and dispersion for RNA-seq data with DESeq2. Genome Biol. 2014;15(12):550.

47. Price MN, Dehal PS, Arkin AP. FastTree 2-approximately maximum-likelihood trees for large alignments. PLoS One. 2010;5(3):e9490.
48. Faith DP. Conservation evaluation and phylogenetic diversity. Biol Conserv. 1992:61:1-10.

49. Gower JC. Generalized procrustes analysis. Psychometrika. 1975;40(1):33-51.

50. Bates D, Maechler M, Bolker B, Walker S. Fitting linear mixed-effects models using Ime4. J Stat Softw. 2015;67(1):1-48.

51. Dixon P. VEGAN, a package of $R$ functions for community ecology. J Veg Sci. 2003;14(6):927-30.

52. Bozdogan H. Model selection and Akaike's Information Criterion (AIC): the general theory and its analytical extensions. Psychometrika. 1987;52:345-70.

53. Benjamini $Y$, Hochberg Y. Controlling the false discovery rate: a practical and powerful approach to multiple testing. J R Stat Soc B. 1995;57(1):289-300.

54. Cook RD. Detection of influential observation in linear regression. Technometrics. 1977;19(1):15-8.

55. Caporaso JG, Kuczynski J, Stombaugh J, Bittinger K, Bushman FD, Costello EK, Fierer N, Pena AG, Goodrich JK, Gordon Jl, et al. QIIME allows analysis of highthroughput community sequencing data. Nat Methods. 2010;7(5):335-6.

56. RDevelopmentCoreTeam. R: a language and environment for statistical computing. Vienna: R Foundation for Statistical Computing; 2008.

57. RStudioTeam. RStudio: Integrated Development for R. RStudio, Inc., Boston, MA. 2015. URL http://www.rstudio.com/.

58. Faust K, Raes J. Microbial interactions: from networks to models. Nat Rev Microbiol. 2012;10(8):538-50.

59. Huson DH, Beier S, Flade I, Gorska A, El-Hadidi M, Mitra S, Ruscheweyh HJ, Tappu R. MEGAN community edition - interactive exploration and analysis of large-scale microbiome sequencing data. PLoS Comput Biol. 2016;12(6):e1004957.

60. Pérez-Losada M, Crandall KA, Freishtat RJ. Comparison of two commercial DNA extraction kits for the analysis of nasopharyngeal bacterial communities. AlMS Microbiol. 2016;2(2):108-19.

61. Mackenzie GA, Leach AJ, Carapetis JR, Fisher J, Morris PS. Epidemiology of nasopharyngeal carriage of respiratory bacterial pathogens in children and adults: cross-sectional surveys in a population with high rates of pneumococcal disease. BMC Infect Dis. 2010;10:304.

62. Cremers AJ, Zomer AL, Gritzfeld JF, Ferwerda G, van Hijum SA, Ferreira DM, Shak JR, Klugman KP, Boekhorst J, Timmerman HM, et al. The adult nasopharyngeal microbiome as a determinant of pneumococcal acquisition. Microbiome. 2014;2:44

63. Allen EK, Koeppel AF, Hendley JO, Turner SD, Winther B, Sale MM Characterization of the nasopharyngeal microbiota in health and during rhinovirus challenge. Microbiome. 2014:2:22.

64. Sakwinska O, Bastic Schmid V, Berger B, Bruttin A, Keitel K, Lepage M, Moine D, Ngom Bru C, Brussow H, Gervaix A. Nasopharyngeal microbiota in healthy children and pneumonia patients. J Clin Microbiol. 2014;52(5):1590-4.

65. Hasegawa K, Mansbach JM, Ajami NJ, Espinola JA, Henke DM, Petrosino JF, Piedra PA, Shaw CA, Sullivan AF, Camargo CA Jr. Association of nasopharyngeal microbiota profiles with bronchiolitis severity in infants hospitalized for bronchiolitis. Eur Respir J. 2016:48(5):1329-339.

66. De Boeck I, Wittouck S, Wuyts S, Oerlemans EFM, van den Broek MFL, Vandenheuvel D, Vanderveken O, Lebeer S. Comparing the healthy nose and nasopharynx microbiota reveals continuity as well as niche-specificity. Front Microbiol. 2017:8:2372

67. Hang J, Zavaljevski N, Yang Y, Desai V, Ruck RC, Macareo LR, Jarman RG, Reifman J, Kuschner RA, Keiser PB. Composition and variation of respiratory microbiota in healthy military personnel. PLoS One. 2017;12(12):e0188461.

68. Yan M, Pamp SJ, Fukuyama J, Hwang PH, Cho DY, Holmes S, Relman DA. Nasal microenvironments and interspecific interactions influence nasal microbiota complexity and S. aureus carriage. Cell Host Microbe. 2013;14(6):631-40.

69. Backhed F, Fraser CM, Ringel Y, Sanders ME, Sartor RB, Sherman PM, Versalovic J, Young V, Finlay BB. Defining a healthy human gut microbiome: current concepts, future directions, and clinical applications. Cell Host Microbe. 2012:12(5):611-22

70. Shade A, Handelsman J. Beyond the Venn diagram: the hunt for a core microbiome. Environ Microbiol. 2012;14(1):4-12.

71. Fu L, Freishtat RJ, Gordish-Dressman H, Teach SJ, Resca L, Hoffman EP, Wang Z. Natural progression of childhood asthma symptoms and strong influence of sex and puberty. Ann Am Thorac Soc. 2014;11(6):939-44.

72. Kwon HL, Ortiz B, Swaner R, Shoemaker K, Jean-Louis B, Northridge ME, Vaughan RD, Marx T, Goodman A, Borrell LN, et al. Childhood asthma and extreme values of body mass index: the Harlem Children's Zone Asthma Initiative. J Urban Health. 2006;83(3):421-33. 
73. Hahn A, Warnken S, Pérez-Losada M, Freishtat RJ, Crandall KA. Microbial diversity within the airway microbiome in chronic pediatric lung diseases. Infect Genet Evol. 2018; (in press).

74. Siegel SJ, Weiser JN. Mechanisms of bacterial colonization of the respiratory tract. Annu Rev Microbiol. 2015;69:425-44.

75. Huang YJ, Nelson CE, Brodie EL, Desantis TZ, Baek MS, Liu J, Woyke T, Allgaier M, Bristow J, Wiener-Kronish JP, et al. Airway microbiota and bronchial hyperresponsiveness in patients with suboptimally controlled asthma. J Allergy Clin Immunol. 2011;127(2):372-381.e1-3.

76. Cardenas PA, Cooper PJ, Cox MJ, Chico M, Arias C, Moffatt MF, Cookson WO. Upper airways microbiota in antibiotic-naive wheezing and healthy infants from the tropics of rural Ecuador. PLoS One. 2012;7(10):e46803.

77. Pérez-Losada M, Graham RJ, Coquillette M, Jafarey A, Castro-Nallar E, Aira M, Freishtat RJ, Mansbach JM. The temporal dynamics of the tracheal microbiome in tracheostomised patients with and without lower respiratory infections. PLoS One. 2017; (in press).

78. Einarsson GG, Comer DM, Mcllreavey L, Parkhill J, Ennis M, Tunney MM, Elborn JS. Community dynamics and the lower airway microbiota in stable chronic obstructive pulmonary disease, smokers and healthy non-smokers. Thorax. 2016;71(9):795-803.

79. National Asthma E, Prevention P. Expert panel report 3 (EPR-3): guidelines for the diagnosis and management of asthma-summary report 2007. J Allergy Clin Immunol. 2007;120(5 Suppl):S94-138.

80. Robinson DS, Campbell DA, Durham SR, Pfeffer J, Barnes PJ, Chung KF, Asthma, Allergy Research Group of the National H, Lung I. Systematic assessment of difficult-to-treat asthma. Eur Respir J. 2003;22(3):478-83.

81. Gallacher DJ, Kotecha S. Respiratory microbiome of new-born infants. Front Pediatr. 2016:4:10

82. Perez GF, Perez-Losada M, Isaza N, Rose MC, Colberg-Poley AM, Nino G. Nasopharyngeal microbiome in premature infants and stability during rhinovirus infection. J Investig Med. 2017;65(6):984-90.

83. Pancham K, Perez GF, Huseni S, Jain A, Kurdi B, Rodriguez-Martinez CE, Preciado D, Rose MC, Nino G. Premature infants have impaired airway antiviral IFNgamma responses to human metapneumovirus compared to respiratory syncytial virus. Pediatr Res. 2015;78(4):389-94.

84. Vissing NH, Chawes BL, Bisgaard H. Increased risk of pneumonia and bronchiolitis after bacterial colonization of the airways as neonates. Am J Respir Crit Care Med. 2013;188(10):1246-52.

85. Bisgaard H, Hermansen MN, Buchvald F, Loland L, Halkjaer LB, Bonnelykke K, Brasholt M, Heltberg A, Vissing NH, Thorsen SV, et al. Childhood asthma after bacterial colonization of the airway in neonates. N Engl J Med. 2007: 357(15):1487-95.

86. Mika M, Mack I, Korten I, Qi W, Aebi S, Frey U, Latzin P, Hilty M. Dynamics of the nasal microbiota in infancy: a prospective cohort study. J Allergy Clin Immunol. 2015;135(4):905-912.e11.

87. Hilton SK, Castro-Nallar E, Perez-Losada M, Toma I, McCaffrey TA, Hoffman EP, Siegel MO, Simon GL, Johnson WE, Crandall KA. Metataxonomic and metagenomic approaches vs. culture-based techniques for clinical pathology. Front Microbiol. 2016;7:484.

88. Bosch AATM, Piters WAAD, van Houten MA, Chu MLN, Biesbroek G, Kool J, Pernet P, de Groot PKCM, Eijkemans MJC, Keijser BJF, et al. Maturation of the infant respiratory microbiota, environmental drivers, and health consequences. Am J Respir Crit Care Med. 2017;196(12):1582-90.

Ready to submit your research? Choose BMC and benefit from:

- fast, convenient online submission

- thorough peer review by experienced researchers in your field

- rapid publication on acceptance

- support for research data, including large and complex data types

- gold Open Access which fosters wider collaboration and increased citations

- maximum visibility for your research: over $100 \mathrm{M}$ website views per year

At BMC, research is always in progress.

Learn more biomedcentral.com/submissions 